\title{
Islam, médias, mise en place du Sénat et article 37 de la Constitution: changement de paradigme au Burkina Faso (1991-2014)?
}

Frédérick Madore

\section{Résumé}

Dans le contexte de libéralisation sociopolitique amorcée depuis 1991 conjugué à la compétition entre les différentes religions dans l'espace public burkinabè, la visibilité de l'islam a accru considérablement avec la création de médias islamiques et l'utilisation croissante de l'internet. Il s'agira ici d'examiner l'impact de la médiatisation croissante de l'islam sur l'agencéité et le contenu des discours des élites musulmanes du Burkina Faso. Elle a conduit à l'émergence de nouvelles figures religieuses très médiatisées qui ont acquis une autorité dans l'espace public. Cependant, la démultiplication du message islamique ne s'est pas accompagnée d'une plus grande politisation. Leur agencéité réside davantage dans leur relative capacité à investir l'espace public pour défendre leur projet de société régie par des normes morales islamiques. Le débat entourant la mise en place du Sénat et la révision de l'article 37 de la Constitution entre 2011 et 2014 a montré l'agencéité en demi-teinte des responsables musulmans.

\section{Introduction}

Depuis les vingt-cinq dernières années, les musulmans du Burkina Faso ont déployé de multiples stratégies pour obtenir une légitimité dans l'espace public. Ceci s'est notamment manifesté par l'ouverture de madâris, ${ }^{1}$ la prolifération de mosquées en milieu urbain - la seule ville de Ouagadougou comptait 632 mosquées en 2008 selon des chiffres de la Fédération des Associations Islamiques du Burkina (FAIB) (Traoré 2010, 29-30) - et un foisonnement associatif avec la présence de plus de 240 associations islamiques membres de la FAIB. L’islam s'est aussi caractérisé par une médiatisation croissante avec la création de médias privés islamiques tels que des périodiques, des stations de radio et une chaine de télévision (Gomez-Perez et Madore 2013; Samson 2011; Savadogo et GomezPerez 2011). À cela il faut ajouter la diffusion d'enregistrements audio de prêches sur différents supports et l'utilisation récente d'internet par les principales associations musulmanes. Ceci témoigne non seulement d'une intensité de la vie religieuse, mais aussi de l'expression de rivalités entre les différentes associations et tendances islamiques (Kouanda 1996), dont le wahhabisme (Cissé 2009; Koné-Dao 2005; Saint-Lary 2012a) et l'Ahmadiyya (Cissé 2010b).

Dans son processus de structuration au cours des premières décennies de l'indépendance, l'islam burkinabè a été secoué par de nombreux remous, voire des crises, autour de différents axes de division notamment entre clans "réformistes", "traditionalistes", "wahhabites", "modernistes", confrériques et ethniques (Kobo 2012; Kouanda 1998; Otayek 1984). Dans ce contexte, l'État a fait en sorte de contrôler la vie religieuse musulmane nationale en intervenant régulièrement dans les divisions internes traversant l'islam burkinabè. Cet "état de subordination politique de l'islam dans l'espace burkinabè" (Otayek 1993, 124) a été d'autant plus prononcé que les instances gouvernementales ont été dominées par une élite christianisée avec l'influence ancienne de l'Église catholique (Otayek 1997) et celle du pentecôtisme depuis le milieu des années 1980 (Laurent 2009). 
Souvent empêtrés dans leurs divisions dogmatiques, les élites musulmanes ont été, dans une grande mesure, absentes des débats sociopolitiques en faisant preuve d'un apolitisme, voire même d'une attitude complaisante envers le pouvoir (Madore 2016) en dépit de certains réveils notamment dans les débats entourant le Projet ZACA (Zone d'aménagement commerciale et administrative) lancé en 2001 (Audet Gosselin 2012; Audet Gosselin et Gomez-Perez 2011). Maud Saint-Lary (2011, 2012b) a souligné quant à elle la montée d'une élite francophone musulmane dans les vingt dernières années, qui participe de manière active à la construction d'un espace public religieux au Burkina Faso et qui prend position sur des enjeux citoyens et sociétaux. Mara Vitale $(\underline{2009}, \underline{2012})$ a pour sa part relevé une redéfinition interne au sein des confréries soufies avec l'affirmation d'une nouvelle génération de chefs religieux burkinabè se posant en "entrepreneur religieux", qui sont moins voués au mysticisme et plus impliqués dans la vie sociale et politique.

Il s'agira ici de s'interroger sur la capacité d'action et d'intervention des élites musulmanes dans les débats sociopolitiques au Burkina Faso dans un contexte de médiatisation croissante de l'islam. Malgré les espaces d'autonomie offerts par l'État depuis l'amorce d'une transition démocratique et d'une libéralisation des télécommunications depuis 1991, les prêcheurs, imams et autres leaders musulmans qui prennent des positions publiques font preuve d'une agencéité en demi-teinte. En effet, si la prolifération des médias islamiques a permis l'émergence de figures d'autorité très visibles dans l'espace public, ceci a toutefois entrainé une pluralisation de l'offre islamique et alimenté des conflits de légitimité religieuse. Ce polycentrisme rendant impossible l'émergence d'une parole autorisée légitime unique s'est accompagné d'une standardisation du contenu des discours. Malgré un apolitisme généralisé, ces acteurs sont tout de même porteurs d'un projet en quelque sorte politique par leur volonté de moralisation et de saturation de l'espace public par le religieux. Cependant, comme les débats entourant la mise en place du Sénat et la révision de l'article 37 de la Constitution entre 2011 et 2014 l'ont montré, le manque de cohérence des élites musulmanes naviguant entre mutisme, complaisance et contestation plus ou moins directe envers le pouvoir conjugué à l'étalement public des divisions internes de la communauté, témoignent des limites de l'agencéité des leaders musulmans et d'un décalage de plus en plus important entre ses élites et sa jeunesse.

Il importe de définir le terme d"“agencéité", qui se veut une des traductions françaises du concept anglophone d'agency. Il ne s'agit pas ici de s'inscrire dans les débats théoriques sur sa conceptualisation ni de développer une sorte de typologie rigoureuse des formes de l'agencéité, mais plutôt d'avoir recours à ce concept comme un outil d'investigation "empirique". Dans un de ses usages les plus communs, l'agencéité est pratiquement synonyme de formes de pouvoir (inégales) que les individus ont à leur disposition pour influencer d'autres personnes ou évènements et de maintenir un certain contrôle dans leurs propres vies. Nous le définissons ici comme l'aptitude d'un agent, d'une personne ou d'une entité à opérer ou à introduire des changements sur les êtres ou sa société de façon consciente ou non, intentionnelle ou non, et ce, sans nécessairement contester le pouvoir ou défaire des normes existantes. $\stackrel{2}{ }$

L'article s'appuie, d'une part, sur des données empiriques tirées d'une enquête de terrain conduite à Ouagadougou à l'automne 2011 et au printemps $2015^{\underline{3}}$ et, d'autre part, sur le dépouillement d'articles de journaux ouagalais. Dans un premier temps, il sera question de la période de la transition démocratique entreprise depuis 1991 afin de mettre en relief les conséquences de la libéralisation sociopolitique sur la visibilité de l'islam et la capacité des responsables musulmans à intervenir dans les débats publics. La seconde partie sera l'occasion de montrer comment des figures musulmanes font preuve d'une certaine agencéité en investissant l'espace public pour défendre leurs projets moraux, dont celui d'une citoyenneté musulmane. Cependant, le cas du débat sur la mise en place du Sénat et 
la révision de l'article 37 de la Constitution entre 2011 et 2014 a été très révélateur de l'agencéité en demi-teinte des élites islamiques au Burkina Faso.

\section{I. "Transition démocratique” et libéralisation de l'espace public: l'islam devant les règles médiatiques}

L'adoption d'une nouvelle constitution en 1991 favorisant une libéralisation politique et sociale ainsi que la forte compétition entre les différentes religions ont fait en sorte que les musulmans aient voulu donner une plus grande visibilité à l'islam. Ainsi, à Ouagadougou, la prolifération de médias islamiques a favorisé l'émergence d'une nouvelle cohorte d'imams et de prêcheurs. Pour certains d'entre eux, il est possible de parler d'une certaine "hypermédiatisation" de leurs propos dans la mesure où leurs discours sont diffusés non seulement dans la sphère religieuse par l'entremise d'une multitude de médias islamiques, mais aussi au-delà des organes religieux où ils sont appelés à se prononcer sur des sujets de société dans certains médias généralistes. Bien que l'offre islamique soit démultipliée et accessible à un plus grand nombre, la plus grande fragmentation de l'autorité religieuse ainsi que la standardisation des discours limitent néanmoins l'agencéité des responsables musulmans.

\section{A. La prolifération des médias islamiques: l'émergence de nouvelles figures médiatisées}

Avec la présentation de la Constitution de la IV République du Burkina Faso le 2 juin 1991, le régime de Blaise Compaoré a entrepris de réintroduire le multipartisme et d'adopter des réformes visant la libéralisation politique et sociale du pays, dont la protection des médias privés. Cette volonté s'est manifestée par la suite avec la création du Conseil Supérieur de l'Information du Burkina Faso (CSI) décrété le $1^{\text {er }}$ août 1995. Puis, par une loi adoptée le 2 février 2005 assurant la constitutionnalisation de l'instance de régulation, le CSI est devenu le Conseil Supérieur de la Communication (CSC). La mise sur pied de cette instance a marqué un tournant dans l'histoire du pays, puisque les différents régimes étatiques depuis l'indépendance s'étaient réservé pratiquement le monopole médiatique. ${ }^{4}$

La libéralisation des télécommunications a accru les possibilités d'expression dans l'espace public. Le pays a alors assisté à une vague de médiatisation spirituelle où la radio, la presse et dans une moindre mesure la télévision et l'internet ont été employés comme voies de prosélytisme et d'affirmation religieuse. Les rassemblements ponctuels dans des lieux publics tels que des hôtels et des stades ainsi que l'utilisation d'affiches et la commercialisation de livres, de films, de cassettes, de CD et de DVD religieux témoignent de la visibilité des groupes chrétiens (Lasseur et Mayrargue 2011; Mayrargue 2004). Ce sont les mouvements (néo)pentecôtistes et catholiques qui se sont initialement montrés les plus entreprenants en créant de nombreux médias confessionnels dès le début des années

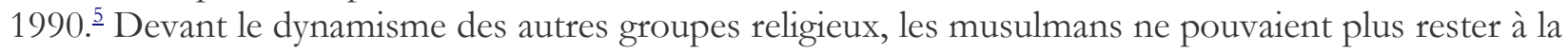
traîne au risque de se voir marginaliser selon plusieurs interlocuteurs rencontrés.

Cette volonté de diversifier les supports de la communication islamique s'est manifestée entre autres par l'apparition de plusieurs périodiques, dont L'Appel, un bimestriel publié entre novembre 1994 et 1999; le bulletin $A n$-Nasr trimestriel depuis 1997 et l'hebdomadaire $A n$-Nasr Vendredi à partir de 2004 par l'Association des Élèves et des Étudiants Musulmans au Burkina (AEEMB) ${ }^{6}$; le CERFIste de 2006 à 2010 par le Cercle d'Études, de Recherches et de Formation Islamiques (CERFI) ${ }^{\text {; }}$; et les mensuels 
islamiques indépendants Al Qibla en 2007 et La Preuve (2007-2011). Actuellement, il n'y a cependant qu'un seul titre visant un public plus large que le milieu associatif et qui est toujours actif: il s'agit du mensuel L'Autre Regard, qui a été lancé en septembre 2012 par Arounan Guigma, qui n'est affilié à aucune association musulmane.

La première radio islamique a, quant à elle, été créée à Bobo-Dioulasso par la Communauté Islamique Ahmadiyya en 2002 - Radio Islamique Ahmadiyya (Samson 2011). La première de Ouagadougou, radio Al Houda, a seulement été lancée en décembre 2004 grâce au financement de la fondation islamique Abdallah Ben Massoud ${ }^{9}$ Elle a été suivie d'une deuxième dans la capitale, Ridwane pour le développement, en mars 2010, sous l'initiative de cheikh Aboubacar Doukouré par le biais de Fondation Islamique Internationale de Charité (FIIC) ${ }^{10}$ dont il a installé la représentation au Burkina Faso. Ouagadougou compte, depuis peu, une troisième radio, IQRA, fondée par le docteur Gaoussou Diawara et Adamé Ouédraogo, surnommé "Karsemba" ("le maitre" en mooré), populaire maitre coranique de la Ligue burkinabè pour la lecture et la mémorisation du Saint Coran (Libulmesco). 11 Il y a eu également la mise sur pied de la chaine de télévision islamique TV Al Houda en juin 2012, elle aussi financée par la fondation islamique Abdallah Ben Massoud. La Communauté Musulmane du Burkina Faso $(\mathrm{CMBF})^{12}$ lancera sous peu une quatrième radio dans la capitale. Le CERFI, quant à lui, travaille actuellement à la rédaction des textes en vue d'une demande auprès du CSC pour obtenir une fréquence pour lancer leur propre radio islamique à moyen ou long terme. ${ }^{13}$

Aux médias plus “traditionnels” s'est ajouté l'internet dans lequel l'islam burkinabè manifeste de plus en plus sa présence bien qu'elle soit encore relativement limitée. En effet, l'AEEMB dispose d'un site web lancé en janvier 2006. ${ }^{14}$ Selon le président de l'AEEMB de l'époque, Tiguiani Nombré, "dans un monde de nouvelles technologies de la communication et de l'information, il est indispensable pour toute organisation sociale d'avoir un site afin de diffuser ses idéaux et d'exprimer sa compréhension". 15 À son avis, ceci est d'autant plus important puisqu" aujourd'hui, bon nombre de sites et de médias diffusent des informations dégradant l'image de l'islam, s’inscrivant ainsi dans la lutte contre les valeurs islamiques. Cette image ne sera pas corrigée si nous n'utilisons pas les mêmes armes que nos ennemis, voire des armes plus performantes." 16 Quant au CERFI,,$^{17}$ à la $\mathrm{CMBF}^{18}$ et au Mouvement Sunnite du Burkina Faso (MSBF), ${ }^{19}$ s'ils disposent eux aussi d'une page web, elles sont cependant mises à jour moins fréquemment, voire plus du tout. De plus, ces sites, incluant celui de l'AEEMB, sont parfois hors ligne, au gré des départs ou mouvements de personnels, ou ne sont plus mis à jour pendant un certain temps.

Par ailleurs, il faut aussi noter la vente de cassettes audio, de CD et de DVD de sermons et prêches dans les kiosques situés devant la Grande mosquée de la CMBF et ailleurs dans la capitale. Des sermons et des prêches de plusieurs figures populaires, telles que le prêcheur Ismaël Derra, l'imam Mohammed Sawadogo, Mohammed Kindo, le grand imam du MSBF, et celui de la CMBF, Aboubacar Sana, sont même disponibles sur internet en téléchargement sur le site web "Le Musulman du Faso", créé en février 2012 et qui a eu, à ce jour, 450000 visites selon les statistiques affichées sur la page d'accueil. ${ }^{20}$ Ces arabisants ont en commun d'être de bons orateurs et d'avoir poursuivi des études islamiques à l'étranger: Derra a entrepris des études en pédagogie islamique en Égypte en tant que

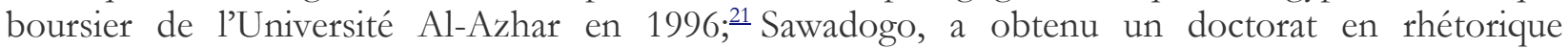
prophétique à l'Université Mohammed Ben Saoud de Riyad en 2001; 22 Kindo, boursier de l'Université

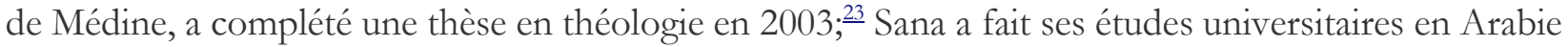
Saoudite dans les années $1970 .{ }^{.4}$ Ces individus, ainsi que quelques autres imams et prêcheurs hautement formés en sciences islamiques, se posent en figures d'autorité, qui sont reconnues pour leurs connaissances islamiques, et ce, devant un auditoire de plus en plus nombreux notamment grâce à leur 
participation dans plusieurs émissions radiophoniques et télévisuelles où sont mis en scène leur savoir religieux et leur éloquence.

Sur la page d'accueil du site web "Le Musulman du Faso", il est aussi possible d'écouter les derniers sermons du vendredi prononcés entre autres à la mosquée de Zangouettin du MSBF et à la Grande mosquée de la CMBF, deux des plus importants lieux de culte de la capitale, ainsi que les prêches hebdomadaires de certaines figures populaires. Dans la section "Les prêches", des enregistrements sont classés par individu ou thèmes. Parmi ceux-ci, "Conseil au fonctionnaire" de Mohammed Sawadogo a été téléchargé à plus de 5700 reprises. Le gestionnaire du site a même numérisé d'anciens prêches de l'imam Aboubacar Sana prononcés entre 1987 et 2003, qui étaient enregistrés sur cassettes audio. Ce site islamique, de loin le plus régulièrement mis à jour au Burkina Faso, est assez complet avec un forum relativement actif, différentes sections thématiques et un flux RSS pour annoncer les titres des dernières informations consultables. Le "Musulman du Faso" a même une présence sur les réseaux sociaux avec sa page Facebook ${ }^{25}$ créée en février 2012, qui a maintenant plus de 1150 mentions “j'aime”. Les utilisateurs peuvent également télécharger une application Android pour téléphones intelligents.

Certaines figures de l'islam burkinabè utilisent de plus en plus les réseaux sociaux pour acquérir une plus grande visibilité. Le jeune cheikh Soufi Moaze Ouédraogo, le guide spirituel de la Communauté Spirituelle Musulmane des Soufis du Burkina Faso (CSMSBF) depuis novembre 2009, est un autre bon


26 juillet 2015. Il ne passe pas inaperçu dans l'espace public lui qui est souvent vêtu de jaune rouge clair avec un turban bleu clair et qui porte des rastas. Ceci lui a d'ailleurs valu le surnom de "Cheikh Rasta". Le journal Le Pays lui a d'ailleurs consacré un article intitulé "Le Cheick qui porte des dreads." "그 Il se sert beaucoup de l'image sur les réseaux sociaux en se mettant en scène dans des photos rendant compte de ses voyages en compagnie de plusieurs savants soufis ou personnalités publiques d'un peu partout à travers le monde. Connaissant le taux d'utilisation de l'internet au Burkina Faso, qui est un des plus faibles dans le monde - seulement 4.4\% de la population burkinabè utilise internet selon des chiffres de juin 2014 et 141740 Burkinabè seraient des utilisateurs de Facebook selon des données de décembre $2012^{28}$, il s'adresse davantage à un profil de jeunes citadins à l'aise avec les nouvelles technologies voire même d'expatriés burkinabè et de fidèles musulmans de partout à travers le monde. La consultation de la liste des individus qui suivent sa page montre qu'un bon nombre d'entre eux ne résident pas au Burkina Faso.

Plusieurs figures parmi les francisants affiliés à l'AEEMB et au CERFI tirent également leur épingle du jeu dans la logique de médiatisation de l'islam. Par leur aisance avec les nouvelles technologies ainsi que leur facilité à communiquer et à présenter un discours en français, certains sont en mesure d'acquérir une visibilité dans les médias francophones. C'est le cas de l'imam Halidou Ilboudo, enseignant de profession, qui anime l'émission hebdomadaire "Foi de croyant" à l'antenne de la chaine de télévision publique RTB depuis février 2011. D’autres, tels que les imams Tiégo Tiemtoré, qui est aussi journaliste, ${ }^{29}$ et Ismaël Tiendrébéogo, qui détient une maitrise en droit et un master en gestion des entreprises à l'Université de Ouagadougou, ${ }^{30}$ sont présents à l'occasion dans la presse privée non confessionnelle.

Ainsi, alors que l'islam burkinabè était généralement considéré comme peu dynamique et en retard par rapport aux autres religions quant à l'utilisation des nouvelles technologies, il répond dorénavant de plus en plus à la tendance de la marchandisation et de la médiatisation du religieux observée à plus grande échelle en Afrique de l'Ouest (Gomez-Perez 2012). La plus grande accessibilité des médias a 
ouvert la voie à de nouvelles figures religieuses de mettre à profit ces outils de communication pour acquérir une légitimité voire une autorité dans l'espace public. Le cas du Burkina Faso s’inscrit dans une dynamique qui s'observe ailleurs dans la sous-région comme au Mali (Holder 2012; Schulz 2003, 2006, 2007, 2012; Soares 2004, 2005), au Niger (Hassane 2009; Sounaye 2011, 2013) et en Côte d'Ivoire (Launay 1997; Savadogo et Gomez-Perez 2011).

S'il est difficile d'évaluer la réception de ces différents médias islamiques et dans quelle mesure ils sont populaires, il n'en demeure pas moins qu'un des objectifs pour les musulmans est d'être présents sur tous les supports et d'inonder l'espace public, qui est devenu un lieu de concurrence religieuse entre entrepreneurs religieux. Si la diffusion de sermons et de prêches sur une variété de médias depuis les vingt dernières années a donné une visibilité sans précédent à plusieurs imams et prêcheurs, tant arabisants que francisants, la plus grande médiatisation de ces acteurs n'a cependant pas entrainé la formulation de discours plus audacieux, mais plutôt une standardisation de leur contenu.

\section{B. D’une médiatisation accrue à une plus grande agencéité? Entre fragmentation et standardisation des discours}

La médiatisation croissante de l'islam burkinabè dans les vingt dernières années a conduit à un paradoxe. D'une part, elle a permis à certaines figures religieuses d'acquérir une grande visibilité médiatique et du même coup, de devenir des acteurs qui ont une influence importante dans l'espace public. La radio notamment favorise la propagation à grande échelle du message islamique et constitue un instrument d'autopromotion efficace pour les figures d'autorité telles qu'Ismaël Derra et Mohammed Kindo en permettant de rejoindre un plus grand bassin d'auditeurs. Outre son accessibilité incomparable - selon des données de 2005, deux tiers des ménages burkinabè disposaient d'une radio alors qu'un sixième d'entre eux avaient une télévision - la radio est capable de "dépasser en partie des clivages pourtant prégnants dans la société burkinabè: rural/urbain; alphabétisé/analphabète; francophone/locuteur des langues nationales" (Capitant 2008, 203). D’autre part, en dépit de rapprochements entre membres de différentes associations, le jeu de concurrence médiatique entre les différentes tendances islamiques et chaines de radios, voire même entre certaines figures animatrices d'émissions religieuses, nuit cependant grandement au consensus musulman. Cette "anarchie" pour reprendre le terme de l'imam Tiégo Tiemtoré, $\underline{31}$ favorise une fragmentation de l'autorité religieuse.

À cela, s'ajoutent les problèmes de ressources financières et humaines touchant l'ensemble des médias islamiques de Ouagadougou. Ceux-ci limitent grandement la qualité du produit et les projets de développement si bien que les trois radios islamiques et la chaine de télévision n’offrent pas une couverture nationale, se limitant à la capitale où elles émettent, car le CSC limite grandement la publicité dans les médias confessionnels et peu de musulmans burkinabè soutiennent leurs radios et télévision. Moussa Sana, le directeur de la Radio Télévision Al Houda, a exposé les difficultés actuelles:

À cause des restrictions financières, on ne peut pas employer beaucoup de gens. Si bien que les gens qu'on a ici, c'est des gens qui font tout en même temps: ils partent filmer, ils reviennent poser leur voix et puis ils montent. Trois choses à la fois.... Le fait qu'on n'a pas beaucoup de ressources, on ne peut pas recruter des gens qui sont vraiment bien formés parce que ça coûte très cher. Donc, on fait avec ce qu'on $a . \underline{32}$

L'agencéité des élites musulmanes est d'autant plus limitée par le fait que la médiatisation de l'islam n'a pas mené à la prononciation de discours plus audacieux, mais s'est plutôt accompagnée d'un contrôle 
de la parole avant diffusion. En effet, les figures religieuses doivent adapter leurs discours de sorte qu'ils respectent les règles inhérentes à la médiatisation prescrites par le CSC. D’une part, l'État a mis en place une législation stricte pour le secteur médiatique. À cet égard, le CSC veille à ce que les radios confessionnelles diffusent un minimum de $20 \%$ de programmes non religieux et qu'elles respectent la laïcité de l'État (Samson 2011). D'autre part, un contrôle étroit est exercé par les radios islamiques sur le contenu diffusé à leurs auditeurs. En ce sens, la majorité des émissions ainsi que les discours des imams et prêcheurs sont enregistrés et présentés en différé. Un responsable de la radio Ridwane a expliqué que l'absence de direct permet de respecter les normes établies et de s'assurer du contenu des diffusions. $\frac{33}{}$ Cette vérification par un comité interne s'observe également à la radio Al Houda (Savadogo et Gomez-Perez 2011).

Il faut dire que le CSC applique assez strictement les règles médiatiques concernant les médias confessionnels et a adressé plusieurs avertissements relativement au principe de tolérance religieuse au cours des dernières années. La RTB télévision et l'imam Ismaël Tiendrébéogo ont reçu une mise en garde pour des propos tenus à l'encontre de la communauté islamique Ahmadiyya dans l'émission "Foi de croyant" diffusée en février 2013..$^{34}$ En octobre de la même année, le CSC a également auditionné les responsables de la chaine de télévision $\mathrm{Al}$ Houda après la diffusion d'une conférence du théologien congolais cheikh Abdoul Madjid Kasogbia en septembre, estimant que certains de ses propos sur le christianisme et le judaïsme violaient plusieurs articles de la convention liant la TV Al Houda au CSC, dont celui de "veiller au respect des différentes sensibilités religieuses." "프 À la suite de cet incident, les responsables de la TV Al Houda ont reconnu leur faute et ils ont pris l'engagement de rendre plus fonctionnel leur comité de visionnage des prêches récemment mis en place et d'initier des formations au profit du personnel pour une autorégulation interne, de manière à éviter les fautes professionnelles. ${ }^{36}$ Plutôt que d'être plus audacieux, le contenu des propos tend vers la standardisation ou la normalisation afin de pouvoir durer dans l'espace médiatique.

La normalisation du contenu des discours se manifeste notamment par la généralisation de l'apolitisme. Une majorité d'imams et de prêcheurs d'une pluralité de tendances ont fait part de l'importance de demeurer dans le cadre religieux et de ne pas verser dans la politique. En fait, bon nombre d'entre eux semblent avoir intégré les règles de l'autocensure. Des questions s'avèrent très délicates et sont évitées comme l'excision ou tout simplement la promulgation d'une loi, car certains sont craintifs d'être victimes de représailles de la part de l'État:

La première chose dont un imam n'abordera pas lors de son sermon du vendredi est de discuter d'une loi. Un imam ne peut pas contester une loi qui a été officiellement adoptée par les politiciens. On peut en discuter en petit groupe, mais pas lors de la prière du vendredi. Si tel est le cas, les autorités pourraient fermer la mosquée.... Si on nous invite à participer, nous prendrons position. Autrement, nous prendrons notre coin et n'en parlerons pas..$^{37}$

Cette autocensure s'inscrit tout particulièrement dans le contexte politique du régime de Compaoré et de son parti, le Congrès pour la Démocratie et le Progrès (CDP). Comme l'ont affirmé Hilgers et Mazzocchetti, la "transition démocratique" au Burkina Faso a débouché sur un "régime de type semiautoritaire" qui, avec des allures de démocratie libérale, s'assure de refermer "le jeu politique au travers de dispositifs informels" (2006, 10-11). La mort de Norbert Zongo, journaliste burkinabè et ancien directeur de publication de l'hebdomadaire L'Indépendant, et trois de ses proches dans un "accident de voiture” le 13 décembre 1998 est un exemple éloquent des dérives autoritaires du pouvoir. ${ }^{38}$ 
En fait, les discours de la très grande majorité des imams et des prêcheurs concernant la politique restent peu engagés. Les responsables de la CMBF, la principale association musulmane du pays, ont toujours été très près du pouvoir ce qui était tout particulièrement le cas de leur ancien président et principal bailleur de fonds, le richissime homme d'affaires Oumarou Kanazoé, décédé en octobre 2011. Celui-ci présidait à la fois la chambre de commerce et d'industrie (novembre 1995-2011), la CMBF (1977-1982; 2004-2011) et la FAIB (2005-2011). Il était régulièrement critiqué par l'opposition en raison de sa proximité avec le régime Compaoré (Cissé 2010a). Cet individu et nombre de commerçants musulmans ont plus ou moins ouvertement travaillé à asseoir les bases sociales du régime et soutenu de façon inconditionnelle le régime Compaoré pour favoriser la prospérité de leurs affaires. Cet apolitisme s'inscrit donc en continuité d'un phénomène qui s'observe dès le début de la période postcoloniale (Cissé 1994; Kouanda 1998; Madore 2016; Otayek 1984). Ceci invite à s'interroger sur l'agencéité des élites musulmanes du Burkina Faso alors que les récents débats sur la mise en place du Sénat et la révision de l'article 37 de la Constitution ont montré une fois de plus les limites de leur capacité d'action.

\section{La moralisation de la société par l'islam: une "citoyenneté musulmane" mise à rude épreuve par l'instauration du Sénat et la révision de l'article 37}

Bien que l'apolitisme et la prudence soient la norme pour bon nombre d'imams, de prêcheurs et de responsables musulmans, les musulmans affiliés à l'AEEMB et au CERFI s'impliquent davantage dans l'arène politique en évoquant les devoirs du citoyen musulman. Si ces acteurs disposent d'une relative capacité à investir l'espace public pour défendre leur projet de société reposant sur des normes morales islamiques tant individuelles que collectives, cette agencéité demeure fragile en raison de la faiblesse de leur rapport de force avec le pouvoir politique. Les débats entourant la mise en place du Sénat et la réforme de l'article 37 de la Constitution illustrent bien les limites de la citoyenneté musulmane. En effet, les silences et les incohérences des représentants musulmans de la FAIB voire même leur participation au clientélisme de l'État en témoignent bien.

\section{A. La moralisation de l'individu et de la société: la figure du "bon musulman"}

Les responsables de l'AEEMB et du CERFI ainsi que leurs imams et prêcheurs, de francisants pour la plupart, dont certains sont des jeunes, se montrent beaucoup plus engagés dans les débats de société que ceux des autres associations. Généralement issus des milieux universitaires francophone et laïc, ils traitent davantage de la chose politique et critiquent parfois l'État. La question du rôle de l'État dans l'organisation du hadj-souvent entachée par des problèmes récurrents (Oubda 2003) - est régulièrement abordée. Une intervention de l'État est parfois encouragée pour réduire les coûts jugés exorbitants reliés à l'accomplissement du pèlerinage. ${ }^{39}$ Le pouvoir est cependant plus souvent critiqué pour son ingérence, vue comme une atteinte à la laïcité. ${ }^{40}$

Leurs représentants n’hésitent généralement pas à commenter les choix importants pour l'électeur musulman. Dans une lettre publiée dans la presse le 28 novembre 2012, le président du CERFI, Moussa Nombo, avait rappelé l'importance des élections législatives et municipales du 2 décembre: 
... Le musulman doit s'engager, et pouvoir éventuellement être candidat pour mieux participer à la gestion de la cité. À tout le moins, le musulman doit élire et faire élire les meilleurs hommes et femmes qui travailleront pour le seul intérêt supérieur de la patrie.... Le CERFI invite chaque Burkinabè à ne ménager aucun sacrifice quand il s'agit de l'avenir commun de notre chère patrie. À cet effet, il faut sortir massivement le 2 décembre prochain pour voter le meilleur candidat.

Cependant, les discours plus politiques se font davantage de façon détournée sans expressément nommer le régime de Compaoré. Ainsi, plutôt que de soutenir ouvertement un ou plusieurs candidats, Moussa Nombo s'en est tenu dans sa lettre à une liste de six critères parmi lesquels se trouvaient l'intégrité et les compétences pour aider les fidèles à faire un choix éclairé. De la même manière, un article du $A n-N a s r V e n d r e d i$ paru en $2010^{42}$ indiquait que les politiciens, sans nommer de noms, devraient s'inspirer du modèle de bonne gouvernance qu'a représenté le règne d'Omar Ibn Abdelaziz. ${ }^{43}$

Les discours des responsables de l'AEEMB et du CERFI mettent surtout l'accent sur l'importance de moraliser la société et d'assainir la culture politique burkinabè. Ainsi, l'agencéité des élites musulmanes se trouve davantage dans leur capacité à se poser comme des guides spirituels influents qui investissent l'espace public pour défendre un projet de société reposant sur les valeurs islamiques. Â l'image de ce qui s'observe ailleurs en Afrique de l'Ouest, nombre d'imams et de prêcheurs débattent des positions de l'islam sur des enjeux sociétaux et édictent des normes et préceptes pour que les musulmans se posent en "sujets moraux" (LeBlanc et Gomez-Perez 2007) aspirant à moraliser leur environnement social. Cette importance accordée à la moralisation de la vie sociale et publique s'observe également dans les autres pays de la sous-région tant du côté de l'islam (Brégand 2009; Holder 2009; Masquelier 1999; LeBlanc 2009, 2012; Saint-Lary et Samson 2011; Samson 2009) que du côté des mouvements pentecôtistes (Mayrargue 2004).

Pour les imams et prêcheurs de l'AEEMB et du CERFI, la moralisation de l'individu a pour modèle la figure du "bon musulman" qui, marquée par une conduite de vie irréprochable, est omniprésente dans leurs discours. Le musulman est exhorté à parfaire ses connaissances afin de mieux pratiquer sa religion. ${ }^{44}$ Celle-ci passe par la manifestation de l'identité islamique dans l'espace public en insistant sur l'importance de la pudeur et de l'hygiène tant corporelle que vestimentaire. ${ }^{45}$ Des articles du $A n-N a s r$ $V$ endredi ainsi que des sermons et des prêches traitent régulièrement de ces questions. Il s'agit d'une véritable entreprise de raffermissement de la foi des croyants (Gomez-Perez et Madore 2013).

La moralisation des individus a aussi une visée collective puisqu'il s'agit non seulement de faire du musulman quelqu'un de plus vertueux selon les préceptes de l'islam, mais aussi d'en faire "un rempart contre les antivaleurs" 46 dans sa société. La figure du bon musulman est ainsi étroitement liée à la bonne pratique citoyenne musulmane pour l'AEEMB et le CERFI. L'islam doit responsabiliser le citoyen-croyant, car la foi musulmane invite le croyant à l'engagement citoyen que ce soit à travers l'implication dans des causes sociales, au sein des ONGs par exemple (Couillard 2013; LeBlanc et al. 2013; Kaag et Saint-Lary 2011), ou dans la participation à la gestion de la cité comme l'a rappelé l'imam Abdoulaye Guitti de l'AEEMB/CERFI dans son sermon du vendredi en avril 2010..$^{47}$ Cependant, bien qu'ils encouragent les bonnes actions citoyennes, ils ne formulent pas concrètement de véritable programme politique.

Malgré tout, comme le rappelle Ismail Salwa (2007), la mise de l'avant d'une identité islamique a des implications politiques. Les projets moraux, tant individuels que collectifs, fondés sur la religion par lesquels les divers domaines de la vie sociale sont investis par des signes et des symboles associés aux 
traditions cultuelles islamiques sont étroitement liés à des projets de réforme de la société. En insistant sur le comportement individuel, plusieurs imams et prêcheurs cherchent à modifier les relations de genre, les normes familiales et d'autres modes de comportement relavant tant de l'espace privé que de l'espace public. La volonté d'introduire des normes islamiques dans ces domaines n'est certes pas nécessairement en lien direct avec la politique étroitement lié à l'exercice du gouvernement et de l'État, mais il s'agit tout de même d'une expression relevant du politique.

Par exemple, l'imam Sana, dans son sermon de l'Ä̈d el-Fitr en août 2013, en avait profité pour fustiger l'homosexualité et l'adoption de lois légalisant le mariage homosexuel par certains pays. Il faut rappeler que la France avait promulgué sa loi du mariage pour tous en mai 2013. L'imam Sana s'était prononcé clairement sur cette question:

Si des autorités décident d'adopter une telle loi, je pense qu'il est temps de leur désobéir. Nous n'accepterons jamais cela. Nous préférons prévenir nos autorités dès maintenant afin qu'elles n'y pensent même pas un instant. Cette loi ne sera pas adoptée dans notre pays. Le respect de l'autorité est une obligation si celle-ci ne fait pas adopter de telles lois dans son pays." $\underline{48}$

Lors de la célébration de la même fête, l'imam Yacoub Tiemtoré de l'AEEMB/CERFI avait lui aussi tenu de tels propos: "Nous notons avec regret et désolation la progression dans le monde, de la légalisation de la perversité à travers le mariage pour tous!" 49

Les imams et les prêcheurs ainsi que certains responsables d'associations musulmanes burkinabè proposent une alternative autant à la tradition qu'à la "modernité occidentale" où l'islam sert à définir un ensemble de normes morales qui constitue une charpente sur laquelle l'individu et le collectif doivent s'appuyer (LeBlanc et Gomez-Perez 2007, 53). Pour ces musulmans, il ne s'agit donc pas de rejeter la modernité, mais de la reconfigurer en l'islamisant selon les principes fondamentaux du Coran. Ceci implique une révolution culturelle dans laquelle les gens deviennent d'honnêtes et vertueux bons musulmans en montrant la voie pour la création d'une nouvelle société. Bien que les imams et prêcheurs se concentrent sur des normes comportementales qui s'inscrivent dans l'espace privé ou public, cela n'aboutit cependant pas véritablement à revoir les fondements de l'État. La question des réformes des institutions politiques l'a bien montré.

\section{B. Les réformes des institutions politiques burkinabè: des associations musulmanes naviguant entre apolitisme, subordination et discordance}

Entre le 7 et le 9 décembre 2011, les assises nationales du Conseil consultatif sur les réformes politiques (CCRP) se sont tenues à Ouagadougou. En tout, 1510 participants ont débattu de différentes questions liées aux réformes démocratiques ce qui a conduit à la remise d'un rapport au président le 21 juillet 2011. Dans celui-ci, la création d'un Sénat a fait l'objet d'une recommandation consensuelle contrairement à la modification de l'article 37 de la Constitution de juin 1991, qui définit les conditions d'éligibilité à l'élection présidentielle. Selon cet article, "Le Président du Faso est élu pour sept ans au suffrage universel direct, égal et secret. Il est rééligible une fois." Cette disposition avait été modifiée en 1997 avec la suppression d"“une fois". Avec la loi modificative du 11 avril 2000, la durée du mandat avait été ramenée à cinq ans et la limitation "une fois" avait été réintroduite. Or, selon un participant du CCRP, "une écrasante majorité” s'est prononcée contre la révision de cet article qui empêchait le président Compaoré de se représenter en 2015. Fait intéressant à noter, les représentants de la chefferie 
traditionnelle et ceux des musulmans se sont abstenus de prendre la parole lors des débats sur l'article 37. ${ }^{50}$ Les musulmans étaient notamment représentés par Adama Sakandé, président par intérim de la

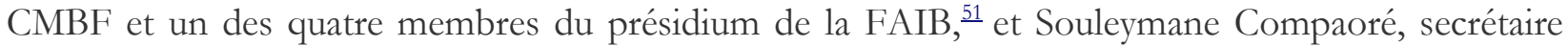
général de la FAIB. En suspendant ainsi leur jugement, les représentants musulmans ont fait preuve une fois de plus d'une frilosité pour les questions relevant de la politique.

Dans la foulée du rapport du CCRP, la mise en place d'un Sénat a été entérinée par l'Assemblée nationale en juin 2012. Cependant, le débat politique sur le bien-fondé d'une telle institution s'est intensifié en 2013 si bien que le 29 juin, des Burkinabè ont protesté sur l'ensemble du territoire national. Les opposants ont fait valoir l'inutilité du Sénat et son budget jugé trop élevé alors que d'autres estimaient que Compaoré avait l'intention de se servir du Sénat pour réviser l'article 37. 52 L'opposition est revenue à la charge lors d'une nouvelle manifestation de quelques dizaines de milliers de participants le 28 juillet 2013, le jour des premières élections sénatoriales. Cependant, devant la grogne populaire, le président a suspendu le processus de mise en place du Sénat et a demandé un réexamen du dossier par le CCRP le 12 août.

L'Église catholique, qui fait régulièrement connaitre sa position sur les évènements et débats majeurs de la vie nationale depuis l'indépendance (Bouron 2011; Couillard 2013; Otayek 1997; Somé 2001), s'est prononcée très clairement contre le Sénat dans une lettre pastorale signée par tous les évêques du pays publiée dans la presse le 21 juillet. .53

De leur côté, les responsables de la communauté musulmane ont fait longtemps preuve de mutisme s'abstenant de donner leur position sur cette question. Lors de la Nuit du destin, célébrée dans la nuit du 4 au 5 août 2013 à la Grande mosquée de Ouagadougou, les dirigeants de la CMBF ont préféré ne pas s'impliquer dans le débat. Adama Sakandé s'est contenté de dire vouloir "prier pour que la paix règne dans notre pays et dans tous les pays musulmans, et que l'entente, entre les enfants de ce pays, se renforce, de même que la cohésion sociale, la solidarité." "uㅜ Quelques jours plus tard, le 8 août 2013, à l'occasion de la prière de l'Ä̈d el-Fitr à la place de la Nation, le grand imam Sana de la CMBF a articulé son sermon autour de l'incivisme ainsi que de la question de l'homosexualité tel que vu précédemment. Il a insisté sur l'importance d'éviter les actes de désobéissances civiles dans le contexte des débats sur

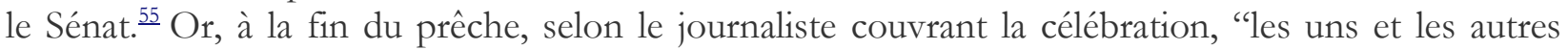
présentaient une mine déconfite, signe que le sermon n'est pas allé au bout des attentes" puisque les gens étaient "impatients de connaître la position de la communauté musulmane sur le Sénat, mais l'imam de la Grande mosquée de Ouagadougou est resté muet sur le sujet". 56 À cet égard, Adama Sakandé a affirmé que les responsables musulmans "s'exprimeront en temps opportun".

De leur côté, l'AEEMB et le CERFI, pour l'Aïd el-Fitr, avaient convié les fidèles pour une conférence publique sur le thème "Islam et civisme: quelles attitudes du musulman face à l'incivisme?". Sans prendre position sur le débat, les intervenants ont appelé gouvernants et gouvernés à parvenir à une cohésion sociale. Selon l'imam Halidou Ilboudo, le mutisme des musulmans face au Sénat peut s'expliquer par le fait qu'ils ne sont pas assez organisés pour parler d'une seule voix au contraire des chrétiens qui peuvent se concerter. ${ }^{58}$ Ces propos illustrent la faiblesse de la FAIB et son incapacité à jouer un rôle de leader, qui est pourtant une fédération regroupant toutes les associations musulmanes. Le sermon de l'Aïd el-Fitr de l'imam Yacoub Tiemtoré, affilié à l'AEEMB et au CERFI, s'est caractérisé par cette même absence de prise de position claire dans le débat en appelant seulement "chaque acteur à des efforts pour la préservation de la paix et du climat social" et aux politiciens de tout horizon politique "d'être mus par l'intérêt général plutôt que par les intérêts partisans ou personnels". $\underline{59}$ 
Cette position de non-intervention des musulmans s'est observée également dans les agissements de la FAIB qui, afin de mieux comprendre la position des deux camps, a rencontré des représentants de l'opposition politique et de la majorité le 22 août 2013. À cette occasion, Souleymane Compaoré a rappelé le statut "de communauté de juste milieu" des musulmans et le fait qu'ils n'avaient pas de position à prendre dans cette querelle: "Nous allons dans l'intérêt général de la Nation. De ce fait, nous avons une position de conciliation, de mise en commun des idées afin de faire évoluer les choses. Notre rôle n'est pas partisan. Nous sommes là pour toutes les forces de la communauté au service de la conciliation entre les camps en présence." $\underline{60}$

Les 13 et 14 septembre, le président Compaoré a rencontré les représentants des différentes communautés religieuses suivant la remise du rapport d'étape circonstancié sur le processus d'opérationnalisation du Sénat, qui a recommandé la présence de représentants des autorités religieuses et coutumières. ${ }^{61}$ L'Église catholique n'est cependant pas revenue sur sa position. Ainsi, dans un communiqué, l'archevêque de Bobo-Dioulasso, Mgr Paul Ouédraogo, a indiqué clairement que l’Église catholique ne siègerait pas au Sénat, car l'Église, "fidèle à sa nature et à sa mission, ne participe pas aux organes délibératifs, exécutifs, législatifs ou judiciaires". $\underline{62}$

Si l'Église catholique a tenu un discours cohérent et sans ambiguités, les représentants de la FAIB ont à l'inverse fait une véritable volte-face. D’une position mitoyenne de “juste milieu” défendue le mois précédent, le secrétaire général de l'association s'est alors posé comme un partisan résolu du projet au terme de son entretien avec le président. Devant la presse, il a défendu le budget de l'institution, "un prix qui ne fait pas peur", pour l'enracinement des institutions. $\frac{63}{}$ Dans la justification de sa position, Souleymane Compaoré a tenu des propos pour le moins contradictoires. D’une part, il a rappelé que la FAIB “n'est pas une organisation politique" et qu'elle n'entend pas être un "contre-pouvoir" et que, par conséquent, les questions politiques doivent être gérées par les politiciens." 64 D'autre part, il a affirmé que les musulmans "trouvent leur intérêt dans le Sénat" et que ces derniers souhaitent y siéger:

Les musulmans sont des citoyens et ils doivent faire de la politique. Ce n'est pas interdit à un religieux de faire de la politique. Déjà vous savez, les musulmans ont participé de tout temps à l'apaisement du climat social quand il y avait des tensions, c'était de la politique.... Ce que les musulmans ne vont pas faire c'est la politique politicienne, être dans les combats alignés derrière des groupes pour des intérêts partisans, ça ce n’est pas possible! $\frac{65}{}$

Ainsi, tout en défendant l'apolitisme de sa fédération, Souleymane Compaoré désirait malgré tout que des représentants musulmans siègent dans un organe délibératif de nature pourtant bien politique. Si cette volte-face peut s'expliquer par les rapports de l'islam burkinabè envers l'État postcolonial historiquement marqués par la subordination, il est plausible de croire que le pouvoir a tenté d'instrumentaliser les autorités musulmanes par le biais de sa contribution de 500 millions francs CFA pour stabiliser le prix du hadj en 2013. De nombreux opposants ont d'ailleurs fait le lien entre la décision prise par les ministères de l'Administration et des Affaires étrangères et le soutien de la FAIB au projet du Sénat ${ }^{66}$ bien que Souleymane Compaoré ait rejeté vigoureusement ces accusations. ${ }^{67} \mathrm{Il}$ est à noter que ce dernier, un ancien journaliste de la RTB, est connu pour être proche du pouvoir de Compaoré. Toujours est-il que sa sortie a surpris les autres représentants de la délégation de la FAIB puisqu'ils s'étaient entendus sur une déclaration écrite devant la presse dans lequel il n'y avait aucune prise de position. $\underline{68}$

L'adhésion claire de la FAIB à la mise en place du Sénat et les nombreuses contradictions de ses responsables ont mis à rude épreuve son capital d'autorité morale et spirituelle. Ceci est d'autant plus 
vrai dans un contexte où le régime de Compaoré devenait de plus en plus critiqué. ${ }^{69}$ D'ailleurs, des internautes musulmans et non-musulmans ont commencé à vilipender les dirigeants de la FAIB dans les sections "commentaires" sur les sites web des différents périodiques burkinabè depuis cette volteface jugeant que les responsables ont été achetés par l'argent du financement du hadj alors que d'autres ont mis en évidence les liens entre le CDP et Souleymane Compaoré. La centaine de commentaires générés par une nouvelle sur l'annonce du soutien de la FAIB au Sénat sur Burkina24 $4^{70}$ témoigne bien de la crise de légitimité de la fédération au sein de la population et des musulmans eux-mêmes.

Le débat sur le Sénat a en outre exacerbé les divisions internes au sein de la communauté dans son ensemble et des associations musulmanes. Depuis 2013, d'intenses débats avaient cours au sein de l'AEEMB et du CERFI quant à la position à adopter concernant la situation nationale. Parmi les responsables, un groupe préférait s'aligner sur la position de la FAIB - et donc d'entretenir le flou comme cela a été le cas lors des différentes sorties publiques des responsables de l'AEEMB et du CERFI - afin de préserver l'union des musulmans et d'éviter de diviser une fois de plus la communauté. Une seconde tendance faisait quant à elle valoir l'autonomie des associations au sein de la fédération et souhaitait donc une prise de position claire. ${ }^{71}$ La sortie controversée de Souleymane Compaoré devant la presse a finalement tranché le débat si bien que l'AEEMB et le CERFI se sont distancés très clairement de la FAIB dans une lettre ouverte signée par les présidents de deux associations et publiée dans les journaux burkinabè le 16 septembre $2013 .{ }^{72}$

Si cette sortie a eu un écho retentissant dans le débat, les responsables de la FAIB avaient déjà fait l'objet de critiques assez acerbes le mois précédent de la part du grand imam du Mouvement Sunnite, Mohammed Kindo, dans son sermon du 20 août 2013. Il avait alors fustigé les dirigeants de la fédération pour leur manque de sincérité et de courage ainsi que leur recherche d'intérêts matériels. ${ }^{73}$ Son sermon, en mooré et n'ayant pas été relayé par la presse généraliste, avait eu moins de répercussions que la déclaration de l'AEEMB et du CERFI.

Du côté de la CMBF, l'association qui, jusqu'alors, ne s'était pas désolidarisée de la FAIB, a vu apparaitre de premières dissensions politiques au sein du bureau exécutif lors du Mouloud le 14 janvier 2014. À l'occasion de cette fête, Assimi Kouanda, le secrétaire exécutif national du CDP et François Compaoré, le frère du président et son conseiller économique, ont annulé à la dernière minute leur déplacement à la Grande mosquée de Ouagadougou gérée par la CMBF pour se rendre plutôt à la mosquée d'Hamdallaye du cheikh Aboubacar Doukouré. ${ }^{74}$ Ils étaient furieux d'apprendre que le Chef de file de l'opposition politique burkinabè (CFOP), Zéphirin Diabré, faisait partie des invités d'honneur de la Grande mosquée. Signe d'un clivage au sein de la CMBF, des responsables de l'association ont fait savoir aux deux politiciens que cette invitation était une initiative personnelle du grand imam Sana, qui n'aurait pas préalablement consulté les membres du bureau exécutif pour obtenir leur accord. ${ }^{75}$

Quelques jours après, François Compaoré aurait appelé l'imam pour le désapprouver. Or, selon l'imam, c'était à la demande du CFOP que Diabré avait été invité à la mosquée la nuit du Mouloud. Voyant plutôt cet acte comme une adhésion de l'imam à l'opposition, François Compaoré et d'autres responsables du CDP ont même engagé des manœuvres auprès du bureau de la CMBF pour destituer le grand imam, mais elles se sont soldées par un échec, devant le soutien de commerçants du grand marché et aux alentours de la Grande mosquée. ${ }^{76}$ Même dans les provinces, des jeunes se disaient prêts à descendre sur la capitale pour le défendre, en signe de refus de le voir démis en coulisses par des manœuvres du pouvoir, en raison de ses prises de position nouvellement critiques envers le CDP. ${ }^{77}$ 
La rupture entre l'imam Sana et le CDP s'est consommée lors de la prière de l'A Äd el-Fitr du 28 juillet 2014 à la place de la Nation à Ouagadougou. Alors que le contexte socio-politique devenait de plus en plus tendu avec la tenue possible d'un référendum pour réviser l'article 37 de la Constitution, le grand imam de Ouagadougou, a commenté l'“inquiétante" situation nationale. Sans mentionner explicitement le référendum sur l'article 37, les fidèles ont bien compris la mise en garde adressée aux acteurs politiques qui souhaitent emprunter cette voie. ${ }^{78}$ Pour les membres du bureau exécutif de l'association, avec les grandes mobilisations de l'opposition et la perte de vitesse du CDP, il valait certainement mieux de quitter le navire que de continuer à soutenir le CDP et risquer de subir la même réprobation des jeunes musulmans. Outre la vague contestatrice née de l'incident du Mouloud de janvier 2014, des signes avant-coureurs s'étaient déjà manifestés en août 2013 lorsque Assimi Kouanda avait été hué à la Grande Mosquée de Ouagadougou lors d'une prière du vendredi. Si cet évènement n'a pas été médiatisé dans la presse, il avait été relayé par les réseaux sociaux.

Les différentes prises de position adoptées par les responsables musulmans tout au long des débats sur le Sénat et l'article 37 ont suscité de nombreuses incompréhensions parmi les Burkinabè et les musulmans eux-mêmes en plus d'alimenter d'importantes divisions internes au sein de la communauté. Au moment de sa création, les initiateurs de la FAIB souhaitaient favoriser la cohésion au sein de la communauté des musulmans et assurer "l'unité d'action des associations islamiques, la désignation d'un porte-parole unique et pour toucher les musulmans et pour le reste du monde". ำ La fédération aura fort à faire pour regagner la confiance de ses membres pour ne pas finir comme une énième tentative des musulmans de se regrouper afin de lutter contre la fitna ${ }^{80}$ après les succès mitigés obtenus par la CMBF et le Conseil Islamique Burkinabè (CIB) créé en 1991.

\section{Conclusion}

La libéralisation politique et sociale du Burkina Faso à partir de 1991 et le contexte religieux concurrentiel ont résulté en une plus grande médiatisation de l’islam. La diffusion de sermons et de prêches sur différents médias a donné une grande visibilité aux discours de plusieurs imams et prêcheurs. Si certains musulmans sont devenus des figures incontournables dans l'espace public en rejoignant des fidèles de plus en plus nombreux, la concurrence entre ces individus et la fragmentation de l'autorité religieuse limitent néanmoins l'agencéité des élites musulmanes. À cela s'ajoute le fait que les discours prononcés par ces figures médiatisées se caractérisent largement par une standardisation du contenu et l'adoption d'un discours plus policé afin de pouvoir perdurer dans l'espace médiatique. Dans le contexte du régime Compaoré, ceci se manifeste également par la généralisation de l'apolitisme et par l'intégration d'une autocensure concernant des questions jugées sensibles.

Les responsables de l'AEEMB et du CERFI se montrent quant à eux beaucoup plus engagés dans les débats de société bien qu'ils ne présentent pas véritablement de programme politique concret. Si l'engagement citoyen est encouragé dans les discours de l'AEEMB et du CERFI, ceci se traduit concrètement surtout par la défense de la moralité. En ce sens, il s'agit d'une forme d'expression politique certes plus indirecte, mais qui n'est pas à exclure. Tout le débat entourant la mise en place du Sénat et la modification de l'article 37 de la constitution a bien illustré les limites de la capacité d'intervention des leaders musulmans dans l'espace public. En effet, la position de grande prudence et de non-intervention adoptée par la FAIB dans un premier temps, puis la défense d'une position traversée d'importantes contradictions dans un second temps ont miné la crédibilité et l'autorité morale des élites musulmanes, qui ont été accusées d'avoir été achetées par l'État par le biais du financement du hadj et d'éventuels sièges au Sénat. Dans la foulée de cette volte-face, l'AEEMB et le CERFI ont 
pris leurs distances de la fédération alors que la $\mathrm{CMBF}$ a connu une crise révélant une fois de plus les divisions internes au sein de l'islam burkinabè et notamment les tensions entre les jeunes et la "vieille garde" musulmane.

Avec la chute du gouvernement Compaoré le 31 octobre 2014 à la suite d'un soulèvement populaire débuté la veille alors que les 127 députés de l'Assemblée nationale devaient de se prononcer sur le projet gouvernemental de révision de la Constitution, il sera intéressant de voir si le nouveau contexte politique va favoriser une reconfiguration de l'islam burkinabè. Certains des membres de la "gérontocratie", qui commande aux destinées de la FAIB et de la CMBF, devront rendre des comptes à la jeunesse musulmane à la suite de leur soutien au régime déchu. Pour bon nombre de jeunes, leurs hauts responsables ont fait preuve d'un manque de leadership, de vision et d'intégrité. Tant à la fédération qu'à la $\mathrm{CMBF}$, de plus en plus de personnes demandent la tenue d'un nouveau congrès pour le renouvèlement des structures exécutives, action qui se fait attendre depuis la mort d'Oumarou Kazanoé en 2011. Or, ce dernier intervenait régulièrement pour apaiser les divisions tout en imposant une chape de plomb sur la conduite de ces associations. Il y a peut-être là une fenêtre d'opportunités pour les jeunes intellectuels musulmans de prendre une plus grande place dans la direction de leur communauté.

\section{Financement}

Cette recherche est financée par le Conseil de recherches en sciences humaines du Canada [No: 767 2012-1819].

\section{Notice biographique}

Frédérick Madore est candidat au doctorat en histoire sous la codirection de Muriel Gomez-Perez (Université Laval) et de Cédric Jourde (Université d'Ottawa). Ses recherches principales portent sur les relations entre la politique et l'islam ainsi que sur le militantisme islamique au Burkina Faso et en Côte d'Ivoire.

\section{Notes}

1. Pluriel de madrasa. Institution d'enseignement où les enfants reçoivent, généralement en arabe, une formation combinant les matières scientifiques et les sciences islamiques, au contraire des écoles coraniques traditionnelles, basées sur la mémorisation du Coran.

2. Sur le concept d'agency, voir notamment Giddens (1979), Taylor (1985), Sewell Jr. (1992), Ahearn

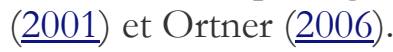

3. Des entretiens ont été conduits auprès de responsables des principales associations islamiques, des membres des médias musulmans, des imams et des prêcheurs.

4. En juin 1984, sous la révolution sankariste (1984-1987), les bureaux du journal indépendant L'Observateur, un des piliers de la presse burkinabé, ont été incendiés. Le journal s'était montré critique envers le pouvoir. 
5. La création de Radio Évangile Développement en 2 juillet 1993, de Radio Maria dans les mois suivants et de Radio Lumière Vie Développement en 1995 en sont de bons exemples.

6. Créée en 1985, cette association vise la promotion de l’islam dans le milieu éducatif francophone laï.

7. Créé en 1989, le CERFI est le prolongement de l'AEEMB. Lorsque les membres n'ont plus le statut d'élève ou d'étudiant et qu'ils se sont insérés sur le marché du travail, ils peuvent devenir membres du Cercle.

8. Anciennement Le vrai visage de l'islam.

9. Le fondateur de cette ONG confessionnelle burkinabé est El Hadj Issouf Kanazoé, un riche commerçant burkinabè. Cette fondation peut notamment compter sur le financement de son président, Mohamed Elsherbini, un Saoudien fortuné. Cette ONG travaille étroitement avec le Mouvement Sunnite, la principale association d'orientation salafiste/wahhabite du pays.

10. Une ONG islamique dont le siège se trouve au Koweït. Sur les différentes ONG confessionnelles impliquées au Burkina Faso, voir notamment LeBlanc et al. (2013).

11. Sur cette figure musulmane et celle du Dr Diawara ainsi que sur la Libulmesco, voir Saint-Lary (2011).

12. Créée en 1962 sous le nom de Communauté Musulmane de Haute-Volta (CMHV), elle se voulait à l'origine comme l'unique association regroupant tous les musulmans au plan national. La création du Mouvement Sunnite en 1973, puis de l'Association Islamique de la Tidjaniyya (AIT) en 1979 ont mis à mal cette volonté. Avant la création de la FAIB en 2005, la CMBF était généralement considérée comme l'association la plus représentative des musulmans du Burkina Faso.

13. Entretien avec Tiégo Tiemtoré, imam de l'AEEMB/CERFI, mosquée de l'AEEMB de Wemtenga, $1^{\text {er }}$ mai 2015.

14. Voir http://www.aeemb.bf.

15. "Association des élèves et étudiants musulmans du Burkina: un site Web, pour plus de visibilité", Sidwaya, 31 janvier 2006.

16. Ibidem.

17. Voir http://www.cerfi.bf.

18. Voir http://cmbf-bf.com. Le site est indisponible depuis quelque temps.

19. Voir http://www.sunna.bf.

20. Voir http://islam.bf.

21. Entretien avec Ismaël Derra, prêcheur, radio Al Houda, 10 octobre 2011.

22. Entretien avec Mohammed Sawadogo, imam, Université Al Houda, 12 octobre 2011. 
23. Entretien avec Mohammed Kindo, imam, radio Al Houda, 22 octobre 2011.

24. "Imam Aboubacar Sana sur l'unité islamique: 'Chez nous, ça patauge dans tous les sens", L'Observateur Paalga, 17 juillet 2014.

25. www.facebook.com/lemusulmandufaso.

26. Voir http://www.facebook.com/cheicksoufi.ouedraogo.

27. "Islam au Burkina: le Cheick qui porte des dreads", Le Pays, 20 novembre 2009.

28. "Internet Usage Statistics for Africa", Internet World Stats, http://www.internetworldstats.com/stats1.htm.

29. Entretien avec Belem Ousmane, militant de l'AEEMB responsable des communications, siège de l'AEEMB, 3 octobre 2011.

30. Entretien avec Ismaël Tiendrébéogo, imam, son bureau, 11 octobre 2011.

31. Entretien avec Tiégo Tiemtoré, imam de l'AEEMB/CERFI, mosquée de l'AEEMB de Wemtenga, $1^{\text {er }}$ mai 2015.

32. Entretien avec Moussa Sana, directeur de la Radio Télévision Al Houda, son bureau, 20 avril 2015.

33. Entretien avec Souleymane Yanogo, responsable de la radio Ridwane, radio Ridwane, 3 octobre 2011.

34. "Rapport public 2013," Conseil Supérieur de la Communication, pp. 2324: http:/ / www.csc.bf/index.php?option=com content\&view=article\&id=316:rapport-public2013\&catid=36: rapports-publics\&Itemid $=118$.

35. Ibid., pp. 20-21.

36. Ibid.

37. Entretien avec Issa Kafando, imam, à sa mosquée, 18 octobre 2011.

38. De nombreux Burkinabè y avaient vu un assassinat orchestré par le régime.

39. Imam Yacoub Tiemtoré, "Sermon de la prière de la Tabaski 2009”, An-Nasr Vendredi, 27 novembre 2009.

40. Imam Ismaël Tiendrébéogo, cité dans "Laïcité: des pistes pour un renforcement du principe juridique", Sidwaya, 26 septembre 2012.

41. "Déclaration du CERFI à l'occasion des élections législatives et municipales couplées du 02 décembre 2012”, Sidwaya, 28 novembre 2012.

42. "Omar ibn Abdel Aziz: un exemple de bonne gouvernance", An-Nasr Vendredi, 1" octobre 2010. 
43. Il a été le huitième calife des Omeyyades (682-720).

44. “Une exigence pour le musulman. Spécial Ramadan: connaissance de l’Islam”, Sidwaya, 7 août 2013.

45. "L'hygiène", An-Nasr Vendredi, 18 novembre 2009; "La pudeur n'apporte que du bien", An-Nasr Vendredi, 7 mai 2010.

46. Imam Tiégo Tiemtoré, "Le Coran et l'éducation", émission Foi de croyant, date inconnue.

47. Imam Abdoulaye Guitti, “La présence citoyenne du musulman,” 23 avril 2010.

48. "Prière de l'Aïd el-Fitr: L'imam de Ouagadougou fustige l'homosexualité," Sidwaya, 8 août 2013;

"Ramadan 2013: La Communauté musulmane contre l'homosexualité", Lefaso.net, 9 août 2013.

49. Imam Yacoub Tiemtoré, "Sermon de la prière de la fête de Ramadan [2013]", http://cerfibf.com/index.php/cerfi-news/22-sermon-de-la-priere-de-la-fete-de-ramadan, page consultée le 19 août 2013.

50. "Réformes politiques: Blaise reçoit le rapport du CCRP le 21 juillet”, L’Observateur Paalga, 14 juillet 2011.

51. Depuis la mort du premier président de la FAIB, Oumarou Kanazoé, en 2011, la présidence de la FAIB est tournante de façon mensuelle entre les présidents des quatre associations fondatrices. En plus d'Adama Sakandé de la CMBF, il y a Aboubacar Doukouré (Ittihad Islami), Aboubacar Maïga II (Communauté Islamique de la Tidjaniyya) et Adama Nikiéma (Mouvement Sunnite).

52. "Journée nationale de protestation: à Ouagadougou, sous la fumée de lacry", L'Observateur Paalga, 30 juin 2013.

53. Cette lettre a notamment été publiée dans le Fasożine et L’Observateur Paalga le 21 juillet et dans Le Pays et Sidwaya le jour suivant.

54. "Nuit du destin: prières pour la paix et la cohésion nationale", Sidwaya, 6 août 2013.

55. "Prière de l'Aïd el-Fitr: l'imam de Ouagadougou fustige l'homosexualité", Sidwaya, 8 août 2013;

"Ramadan 2013: La Communauté musulmane contre l'homosexualité", Lefaso.net, 9 août 2013.

56. “Aid el Fitr 2013: Le gage de l’imam Sana pour la paix”, L’Observateur Paalga, 11 août 2013.

57. Ibidem.

58. "Nuit du destin: la question du civisme au cœur de la commémoration", Sidwaya, 6 août 2013; "Nuit du Destin: la situation nationale s’invite au débat”, Le Pays, 6 août 2013.

59. Imam Yacoub Tiemtoré, "Sermon de la prière de la fête de Ramadan [2013]", http://cerfibf.com/index.php/cerfi-news/22-sermon-de-la-priere-de-la-fete-de-ramadan, page consultée le 19 août 2013.

60. "Mise en place du Sénat: la Communauté musulmane veut mieux comprendre la position des différentes parties", Lefaso.net, 23 août 2013. 
61. “Rapport sur le Sénat: voici le document officiel”, L’Observateur Paalga, 3 septembre 2013.

62. "Participation au Sénat: le niet de l’Église catholique”, L’Observateur Paalga, 15 septembre 2013.

63. "Sénat: la communauté musulmane soutient la mise en place de l'Institution", Burkina 24, 14 septembre 2013.

64. "Les musulmans partants, les protestants dans l'expectative. Mise en place du Sénat", Sidwaya, 15 septembre 2013 .

65. "Les catholiques rejettent, les musulmans bénissent et les protestants... ”, L’Observateur Paalga, 15 septembre 2013.

66. "Dieu, Allah et les 'coutumiers' appelés à rescousse du projet de Sénat au Burkina Faso", Lefaso.net, 20 septembre 2013.

67. "Mise en place du Sénat: la communauté musulmane donne son aval”, Sidwaya, 14 septembre 2013.

68. Entretien avec Halidou Ilboudo, imam de l'AEEMB/CERFI, son bureau, 14 avril 2015; entretien avec Dr Aboubacar Doukouré, membre du présidium de la FAIB, à sa résidence, 15 avril 2015.

69. Le parti du président a été victime d'une vague de démissions sans précédent le 4 janvier 2014 avec le départ de Roch Marc Christian Kaboré, Salif Diallo et Simon Compaoré, des ténors du régime Compaoré, pour fonder leur propre parti, le Mouvement du Peuple pour le Progrès (MPP).

70. Voir http://http://burkina24.com/news/2013/09/14/senat-la-communaute-musulmanesoutien-la-mise-en-place-de-linstitution.

71. Entretien avec Abdoulaye Ly, journaliste et ancien militant de l'AEEMB et du CERFI, son bureau, 22 avril 2015.

72. "Sénat: l'AEEMB et le CERFI se démarquent des propos de Souleymane Compaoré", Lefaso.net, 16 septembre 2013.

73. "Les Burkinabè et le Sénat: pourquoi la division des musulmans?", Le vrai visage de l'islam, n 8,5 octobre au 5 novembre 2013.

74. Depuis la fin des années 1980, Doukouré, grâce à ses accointances avec le monde arabe, est devenu un des proches conseillers du président du Burkina Faso concernant les relations avec les pays arabomusulmans.

75. "François et Assimi veulent la tête de l'Imam Sana”, Mutations, $1^{\text {er }}$ août 2014.

76. Ibid.

77. Entretien avec Abdoulaye Ly, journaliste et ancien militant de l'AEEMB et du CERFI, son bureau, 22 avril 2015.

78. “Ramadan 2014: L’imam Sana ou la pédagogie de l’horreur”, L’Observateur Paalga, 29 juillet 2014. 
79. "Associations islamiques du Burkina: une seule voix, un seul 'muezzin", L’Observateur Paalga, 15 décembre 2005.

80. Ce mot arabe réfère aux désaccords et aux divisions parmi les musulmans.

\section{Bibliographie}

Ahearn, Laura M. 2001. "Language and Agency." Annual Review of Anthropology 30 (1): 109137.10.1146/annurev.anthro.30.1.109.

Audet Gosselin, Louis. 2012. Le projet ZACA. Marginalisation, résistances et reconfigurations de l'islam à Onagadongou, 2001-2006. Québec: Presses de l’Université Laval.

Audet Gosselin, Louis, et Muriel Gomez-Perez. 2011. "L'opposition au projet ZACA à Ouagadougou (2001-03): feu de paille ou mutations profondes de l'islam burkinabè?" Revue canadienne des études africaines 45 (2): 273-309.

Bouron, Jean-Marie. 2011. “Amitiés, inimitiés. Les rapports incertains de l’Église catholique avec la Première République voltaïque (1960-1966).” Civilisations 60 (1): 123-142.

Brégand, Denise. 2009. "Les réformistes et l'État au Bénin.” In Islam, État et Société en Afrique, sous la direction de René Otayek et Benjamin Soares, 187-210. Paris: Karthala.

Capitant, Sylvie. 2008. "La radio en Afrique de l'Ouest, un “média carrefour” sous-estimé ? L'exemple du Burkina Faso.” Réseaux 150 (4): 189-217.

Cissé, Issa. 1994. "Islam et État au Burkina Faso: de 1960 à 1990." Thèse de doctorat en histoire, Université de Paris VII - Denis Diderot.

Cissé, Issa. 2009. "Le wahhabisme au Burkina Faso: dynamique interne d'un mouvement islamique réformiste." Cabiers du CERLESHS 33: 1-33.

Cissé, Issa. 2010a. "El hadj Oumarou Kanazoé: homme d'affaires et mécène dans la communauté musulmane burkinabè." Cahiers du CERLESHS 34: 151-186.

Cissé, Issa. 2010b. “La Ahmadiyya au Burkina Faso.” Islam et sociétés au sud du Sahara 2: 95-116.

Couillard, Kathéry. 2013. "Action sociale et espace public: l'Église catholique et les associations musulmanes à Ouagadougou (Burkina Faso) (1983-2010)." Mémoire de maitrise en histoire, Université Laval, Québec.

Giddens, Anthony. 1979. Central Problems in Social Theory: Action, Structure, and Contradiction in Social Analysis. Berkeley: University of California Press.

Gomez-Perez, Muriel. 2012. "Political Islam in West Africa From the 1980s to the Present." In Whatever Happened to the Islamists? Salafis, Heavy Metal Muslims and the Lure of Consumerist Islam, sous la direction de Amel Boubekeur et Olivier Roy, 129-152. London: Hurst.

Gomez-Perez, Muriel, et Frédérick Madore. 2013. "Prêcheurs(ses) musulman(e)s et stratégies de communication au Burkina Faso depuis 1990. Des processus différentiés de conversion interne." Théologiques 21 (2): 121-157.10.7202/1028465ar 
Hassane, Souley. 2009. "Société civile islamique: esquisse sur l'islam postmoderne et les pratiques religieuses "globales" en Afrique." In L'islam, nouvel espace public en Afrique, sous la direction de Gilles Holder, 101-125. Paris: Karthala.

Hilgers, Mathieu, et Jacinthe Mazzocchetti. 2006. "Introduction au thème. L'après Zongo: entre ouverture politique et fermeture des possibles." Politique africaine (101): 518.10.3917/polaf.101.0005

Holder, Gilles. 2009. “Maouloud 2006”, de Bamako à Tombouctou. Entre réislamisation de la nation et laïcité de l'État: la construction d'un espace public religieux au Mali." In L'islam, nouvel espace public en Afrique, sous la direction de Gilles Holder, 237-289. Paris: Karthala.

Holder, Gilles. 2012. "Chérif Ousmane Madani Haïdara et l'association islamique Ançar Dine: un réformisme malien populaire en quête d'autonomie." Cabiers d'études africaines 206-207 (2): 389-425.

Kaag, Mayke, et Maud Saint-Lary. 2011. "Nouvelles visibilités de la religion dans l'arène du développement. L'implication des élites chrétiennes et musulmanes dans les politiques publiques en Afrique." Bulletin de l'APAD (33).

Kobo, Ousman M. 2012. Unveiling Modernity in Twentieth-Century West African Islamic Reforms, Islam in Africa. Leiden: Brill.10.1163/9789004233133

Koné-Dao, Maïmouna. 2005. "Implantation et influence du wahhâbisme au Burkina Faso de 1963 à 2002." In L'islam politique an sud du Sahara: identités, discours et enjeux, sous la direction de Muriel Gomez-Perez, 449-459. Paris: Karthala.

Kouanda, Assimi. 1996. "La lutte pour l'occupation et le contrôle des espaces réservés aux cultes à Ouagadougou." In Le Burkina entre révolution et démocratie (1983-1993), sous la direction de René Otayek, Filiga Michel Sawadogo et Jean-Pierre Guingané, 91-99. Paris: Karthala.

Kouanda, Assimi. 1998. "Les conflits au sein de la Communauté musulmane du Burkina: 1962-1986." In Islam et islamismes au sud du Sahara, sous la direction de Ousmane Kane et Jean-Louis Triaud, 83100. Paris: Karthala.

Lasseur, Maud, et Cédric Mayrargue. 2011. "Introduction au thème. Le religieux dans la pluralisation contemporaine: éclatement et concurrence.” Politique africaine (123):5-25.10.3917/polaf.123.0005

Launay, Robert. 1997. "Spirit Media: The Electronic Media and Islam among the Dyula of Northern Côte d'Ivoire." Africa: Journal of the International African Institute 67 (3):441-453.10.2307/1161183

Laurent, Pierre-Joseph. 2009. Les pentecôtistes du Burkina Faso: mariage, pouvoir et guérison. Paris: Karthala.

LeBlanc, Marie Nathalie. 2009. "Foi, prosélytisme et citoyenneté culturelle: le rôle sociopolitique des jeunes arabisants en Côte d'Ivoire au tournant du XXI ${ }^{\mathrm{e}}$ siècle." In L'islam, nouvel espace public en Afrique, sous la direction de Gilles Holder, 173-196. Paris: Karthala.

LeBlanc, Marie Nathalie. 2012. "Du militant à l'entrepreneur: les nouveaux acteurs religieux de la moralisation par le bas en Côte-d'Ivoire." Cahiers d'etudes africaines 206-207 (2): 493-516.

LeBlanc, Marie Nathalie, et al. 2013. "Les ONG confessionnelles en Afrique de l'Ouest: un équilibre précaire entre prosélytisme et professionnalisme au Burkina Faso." Canadian Journal of Development Studies/Revue canadienne d'études du développement 34 (2): 236-256.10.1080/02255189.2013.791259

Leblanc, Marie Nathalie, and Muriel Gomez-Perez. 2007. "Jeunes musulmans et citoyenneté culturelle: retour sur des expériences de recherche en Afrique de l'Ouest francophone." Sociologie et sociétés 39 (2): 39-59.10.7202/019083ar 
Madore, Frédérick. 2016. La construction d'une sphère publique musulmane en Afrique de l'Ouest. Québec, Presses de l'Université Laval.

Masquelier, Adeline. 1999. "Debating Muslims, Disputed Practices: Struggles for the Realization of an Alternative Moral Order in Niger." In Civil Society and the Political Imagination in Africa: Critical Perspectives, sous la direction de John L. Comaroff et Jean Comaroff, 219-250. Chicago, IL: University of Chicago Press.

Mayrargue, Cédric. 2004. "Trajectoires et enjeux contemporains du pentecôtisme en Afrique de l’Ouest." Critique internationale 22 (1): 95-109.10.3917/ crii.022.0095

Ortner, Sherry B.2006. Anthropology and Social Theory: Culture, Power, and the Acting Subject. Durham: Duke University Press.10.1215/9780822388456

Otayek, René. 1984. "La crise de la communauté musulmane de Haute-Volta. L'islam voltaïque entre réformisme et tradition, autonomie et subordination." Cabiers d'études africaines 24 (95): 299_ 320.10.3406/cea.1984.2205

Otayek, René. 1993. "Une relecture islamique du projet révolutionnaire de Thomas Sankara." In Religion et modernité politique en Afrique noire: Dieu pour tous et chacun pour soi, sous la direction de JeanFrançois Bayart, 101-127. Paris: Karthala.

Otayek, René. 1997. "L’Église catholique au Burkina Faso. Un contre-pouvoir à contretemps de l'histoire?" In Religion et transition démocratique en Afrique, sous la direction de François Constantin et Christian Coulon, 221-258. Paris: Karthala.

Oubda, Mahamoudou. 2003. L'islam au Burkina Faso. Problématique de l'organisation du badj. Ouagadougou: Centre africain de diffusion islamique et scientifique.

Saint-Lary, Maud. 2011. Le Coran en cours du soir. La formation comme outil de réislamisation des musulmans francophones. Ethnographiques.org 22. www.ethnographiques.org/2011/Saint-Lary.

Saint-Lary, Maud. 2012a. "Du wahhabisme aux réformismes génériques: renouveau islamique et brouillage des identités musulmanes à Ouagadougou." Cabiers d'études africaines 206-207 (2): 449_ 470.

Saint-Lary, Maud. 2012b. "Quand le droit des femmes se dit à la mosquée: ethnographie des voies islamiques d'émancipation au Burkina Faso.” Autrepart 61 (2): 137-155.10.3917/autr.061.0137

Saint-Lary, Maud, et Fabienne Samson. 2011. Pour une anthropologie des modes de réislamisation. Supports et pratiques de diffusion de l'islam en Afrique subsaharienne. Ethnographiques.org 22. www.ethnographiques.org/2011/Saint-Lary,Samson\#nh4.

Salwa, Ismail. 2007. "Islamism, Re-Islamisation and the Fashioning of Muslim Selves: Refiguring the Public Sphere.” Muslim World Journal of Human Rights 4 (1):article 3.

Samson, Fabienne. 2009. "Nouveaux marabouts politiques au Sénégal. Lutte pour l'appropriation d'un espace public religieux." In L'islam, nouvel espace public en Afrique, sous la direction de Gilles Holder, 149-172. Paris: Karthala.

Samson, Fabienne. 2011. "La guerre des ondes comme mode de prosélytisme. La Ahmadiyya et les médias au Burkina Faso." Ethnographiques.org 22. www.ethnographiques.org/2011/Samson\#nh10.

Savadogo, Mathias, et Muriel Gomez-Perez. 2011. La médiatisation des prêches et ses enjeux. Regards croisés sur la situation à Abidjan et à Ouagadougou. Ethnographiques.org 22. www.ethnographiques.org/2011/Savadogo,Gomez-Perez. 
Schulz, Dorothea E. 2003. "“Charisma and Brotherhood' Revisited: Mass-Mediated Forms of Spirituality in Urban Mali." Journal of Religion in Africa 33 (2): 146-171.10.1163/15700660360703123

Schulz, Dorothea E. 2006. "Promises of (Im)mediate Salvation: Islam, Broadcast Media, and the Remaking of Religious Experience in Mali." American Ethnologist 33 (2): 210229.10.1525/ae.2006.33.2.210

Schulz, Dorothea E. 2007. "Evoking Moral Community, Fragmenting Muslim Discourse: Sermon Audio-recordings and the Reconfiguration of Public Debate in Mali." Journal for Islamic Studies 27: 39-72.

Schulz, Dorothea E. 2012. Muslims and New Media in West Africa: Pathways to God. Bloomington: Indiana University Press.

Sewell Jr., William H. 1992. "A Theory of Structure: Duality, Agency and Transformation.” American Journal of Sociology 98 (1): 1-29.10.1086/ajs.1992.98.issue-1

Soares, Benjamin F. 2004. "Islam and Public Piety in Mali." In Public Islam and the Common Good, sous la direction de Armando Salvatore et Dale F. Eickelman, 205-226. Leiden: Brill.

Soares, Benjamin F.2005. Islam and the Prayer Economy: History and Authority in a Malian Town. Edinburgh: Edinburgh University Press.10.3366/edinburgh/9780748622856.001.0001

Somé, Magloire. 2001. "Christian Base Communities in Burkina Faso: Between Church and Politics.” Journal of Religion in Africa 31 (3): 275-304.10.1163/157006601X00185

Sounaye, Abdoulaye. 2011. La "discothèque" islamique: CD et DVD au cœur de la réislamisation nigérienne. Ethnographiques.org 22. http://www.ethnographiques.org/2011/Sounaye.

Sounaye, Abdoulaye. 2013. "Alarama is all at Once: Preacher, Media "Savvy", and Religious Entrepreneur in Niamey." Journal of African Cultural Studies 25 (1): 88102.10.1080/13696815.2012.749782

Taylor, Charles. 1985. Human Agency and Language, Philosophical Papers. Cambridge: Cambridge University Press.10.1017/CBO9781139173483

Traoré, Bakary. 2010. "À la recherche d’une voie africaine de la laïcité. Islam et pluralisme religieux au Burkina Faso." In Islam et sociétés au sud du Sahara, sous la direction de Jean-Louis Triaud, 954. Paris: Rivages des Xantons.

Vitale, Mara. 2009. "Économie morale, islam et pouvoir charismatique au Burkina Faso." Afrique contemporaine 231 (3): 229-243.10.3917/afco.231.0229

Vitale, Mara. 2012. "Trajectoires d'évolution de l'islam au Burkina Faso.” Cabiers d'études africaines 206207 (2): 367-387. 